\title{
Case Report on Binder Syndrome
}

\section{Prishni Devi Dutta ${ }^{1 *}$ and Swagata Khanna ${ }^{2}$}

${ }^{1}$ Junior Consultant, Department of ENT and Head and Neck Surgery, Swagat Super

Speciality Surgical Institute, Guwahati, Assam, India

${ }^{2}$ Chief Consultant and Professor, Department of ENT and Head and Neck Surgery,

Swagat Super Speciality Surgical Institute, Guwahati, Assam, India

*Corresponding Author: Prishni Devi Dutta, Junior Consultant, Department of ENT and Head and Neck Surgery, Swagat Super Speciality Surgical Institute, Guwahati, Assam, India.
Received: July 03, 2021

Published: July 22, 2021

(C) All rights are reserved by Prishni Devi

Dutta and Swagata Khanna.

\section{Abstract}

Binder syndrome or Maxillonasal dysplasia is a congenital malformation. Presenting a case report of 15 year old patient with history of flat nose, who was unhappy with his appearance. He underwent augmentation rhinoplasty and conchal and rib cartilage was used for reconstruction. The surgery was aimed at correction of nasal deformity focusing the patients main complain, to increase the dorsum and tip.

Keywords: Binder Syndrome; Maxillonasal Dysplasia; Congenital Malformation

\section{Introduction}

Binder syndrome or Maxillonasal dysplasia is a congenital malformation. It was first described by Von Binder in 1962 as a syndrome of flat bridge, absent frontonasal angle, absent anterior nasal spine, short columella, acute naso labial angle, convex upper lip and grade 3 malocclusion.

He postulated these defects were caused by rhinocephalic dysplasia which he called Maxillonasal. Dysostosis [1].

Inheritance: It is sporadic in majority of the cases $[1,2,4]$.

Familial recurrence has been noted by number of authors. Horswell., et al. found dominant inheritance with reduced penetrance in some pedigrees. Olon-Nordenram took a formal genetic analysis and suggested that recurrence could be explained by autosomal recessive inheritance with reduced penetrance $[3,5]$.

Etiology: Though different theories have been suggested, the most accepted one is the disturbance of prosencephalic induction centre during embryonic growth [1-3].
Birth trauma has been cited as a possible causative factor but has not been substantiated in literature $[1,3]$.

Teratogenic exposure to agents causing vitamin K deficiency like lithium, ethanol and or therapeutic use of warfarin and phenytoin has been seen in some studies $[3,4]$.

The most striking features are arhinoid face with mid face hypoplasia, unusually flat vertical nose due to abnormal position of nasal bones, broad philtrum, perpendicular alar cheek junction, lack of tip projection, and crescent shaped alae.

The perialar flatness in cephalometric studies have showed is due to a small maxilla positioned posteriorly on a short anterior cranial base [4].

In binders syndrome there is acute nasolabial angle which is 76 - 88 degree (Holmstoem., et al.) instead of normal 103 - 110 degree due to convexity of upper lip. Other features seen are anterior nasal spine agenesis [1]. 
These patients also have relative prognathism and type 3 malocclusion. But however most of the patients have acceptable occlusion with no or very little need for orthodontic treatment. Besides these some may show hypoplastic or absent frontal sinuses (40 $50 \%$ ) and about $44.2 \%$ present with irregularities in cervical spine as there is a common concurrent induction process for both the prosencephalic area and vertebrae $[1,3]$.

\section{Case History}

A 15 year male patient reported to OPD with chief complain of facial deformity. He has been visiting many times since 2 - 3 years for his growing concern about his appearance. No family history of similar complaint, and medical history was non-contributory.

External examination of nose- He had a broad, flat nose and dorsum with no tip support, b/l flat alae, broad philtrum and convex upper lip.

Intra oral examination - normal dentition. The functional status of nose and paranasal sinus was normal.

Investigations: Routine investigations, preoperative photographs, $\mathrm{X}$ ray nasal bone lateral view.

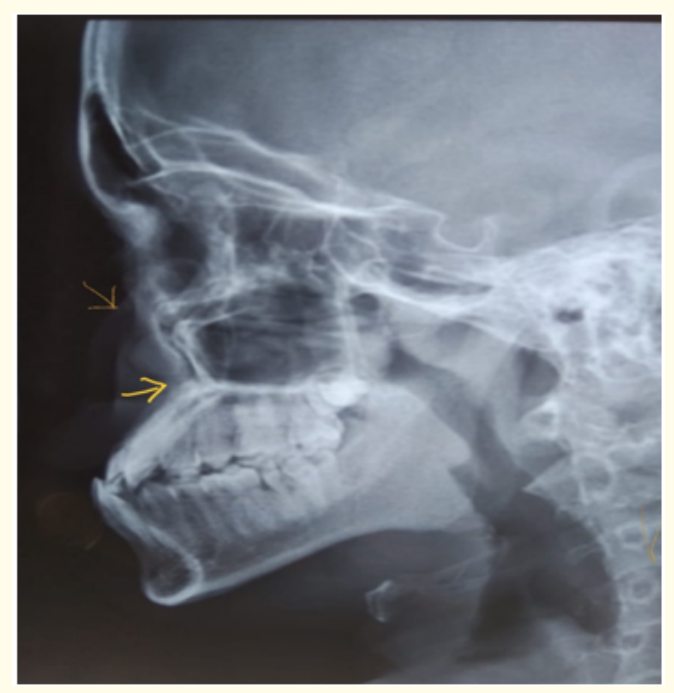

Figure 1: Lateral $x$ ray of nose-showing the absent anterior nasal spine and maldeveloped nasal bone (yellow arrows).

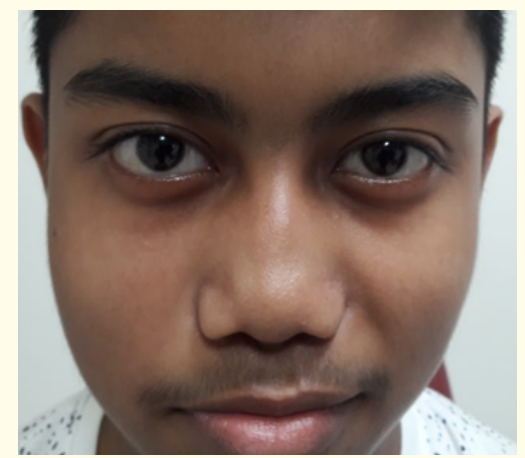

Figure 2: Preoperative picture. Frontal view.

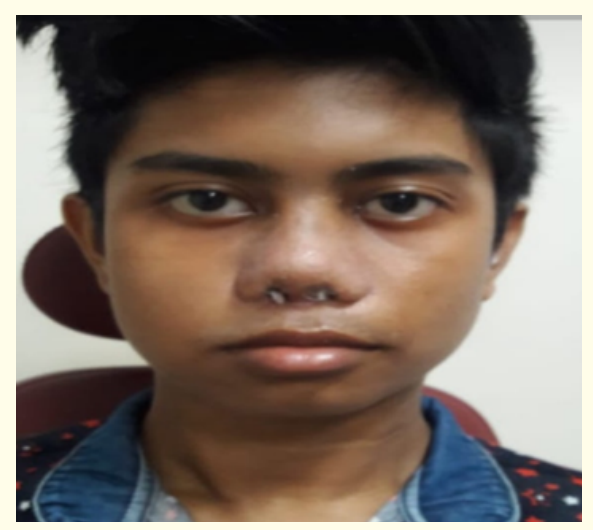

Figure 3: Post-operative picture. Frontal view.

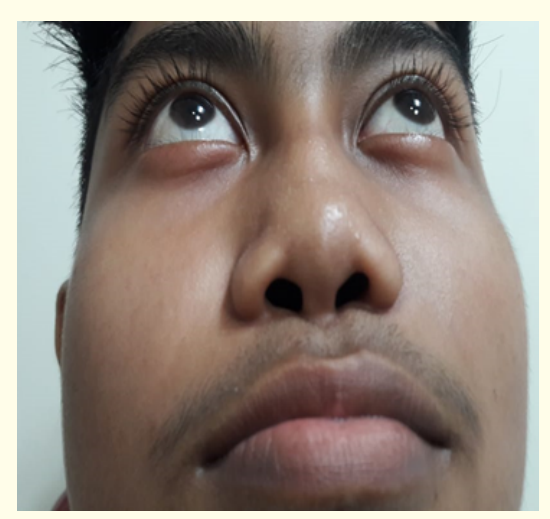

Figure 4: Basal view (Pre-operative). 


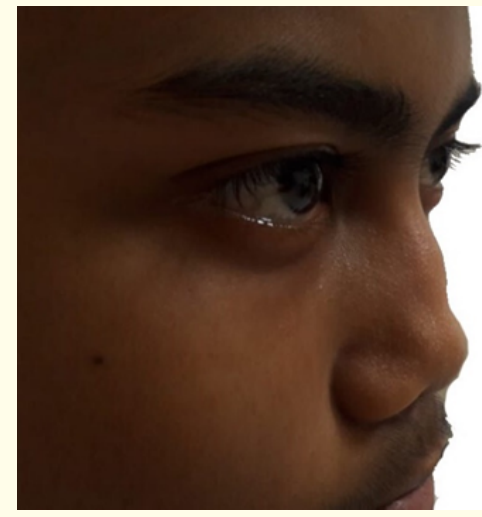

Figure 5: Lateral view (Pre-operative).

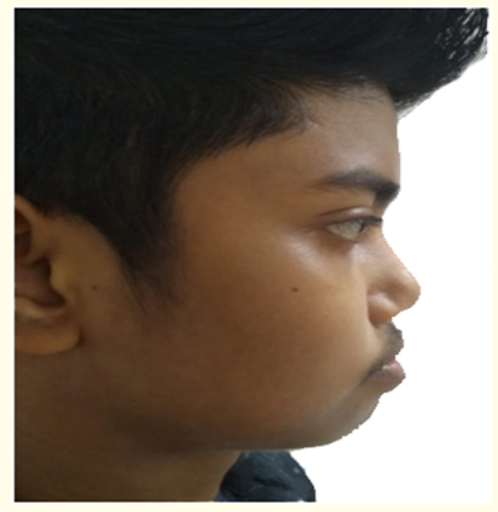

Figure 6: Lateral view (Post-operative 4 weeks).

Although normally rhinoplasty is considered in adult patients but in our case as the patient insisted, the surgery was done early. The patient was also explained about the procedure, and consent for cartilage harvesting was taken and informed that he might need a second surgery also.

Procedure: Under GA, augmentation rhinoplasty with external approach was done. Both rib and conchal cartilage was harvested for the reconstruction A midcolumellar incision was given. The nasal dorsum and tip was reconstructed with $6^{\text {th }}$ rib cartilage (right) harvested.

The dorsal augmentation was done with dorsal onlay graft and columellar cartilage strut was used for tip support.
A Spreader graft with costal cartilage for widening the nasal valve was used.

As Alae was found to be thin and mal developed intraoperatively, bilateral alar reconstruction was done with autologous conchal cartilage (right). Small piece of cartilage was placed in subcutaneous pocket between upper lip for correction of acute naso-labial angle and to narrow the philtrum.

\section{Discussion}

Patients with midface hypoplasia due to their clinical appearance need surgical and orthodontic treatment. Management depends on level of complexity because of variation in midface discrepancy and occlusal relationship. Usually, they can require more than one surgical procedure due to graft resorption and an unsatisfactory appearance.

Hence factors like age, patients psychology and understanding, probable need for further procedures in such patients must be taken into consideration.

In this case, the surgery was done early due to repeated request from patient and his parents as he was constantly being ridiculed for his flat nose.

The surgery was aimed at correction of nasal deformity focusing the patients main complain, to increase the dorsum, allowing tip projection and support, and increase the nasal length.

Generally mild cases without severe malocclusion can be managed by rhinoplasty like using cartilage graft around dorsum and tip, correcting the paranasal and infraorbital deformity without osteotomy as like this case $[4,5]$.

Both bone and cartilage use has been mentioned in literature according to surgeons need. Among the grafts - bone subject to remodeling but risk of resorption is there. Cartilage have a low rate of infection, easy to shape and has natural feel in-situ (Gewalli, Berlanga., et al. 2008). However, rib cartilage have a tendency to warp which alters configuration of cartilage graft over time and graft irregularities may be visible through skin as well [5].

First attempt may not be successful and may require revision rhinoplasty to get an acceptable nose and appearance. 


\section{Conclusion}

Though binder syndrome is a not a disease per se, but a facial deformity is considered difficult and challenging case by any rhinoplasty surgeon.

As the surgery was done before completion of complete growth of nasal structures, he might need revision rhinoplasty if need arises. Mostly correction of the facial deformity and reconstruction in these patients is considered to relieve them of the psychological stress they face.

\section{Bibliography}

1. Jain U., et al. "Binders syndrome -Rare disease". BMJ Case Report 10 (2011): 1-4.

2. Gewalli F., et al. "Nasomaxillary Reconstruction in binders syndrome: Bone vs cartilage grafts-A long term intercentre comparison between Sweden and Mexico". Journal of Craniofacial Surgery 19.5 (2007): 1225-1236.

3. Quarell O., et al. "Maxillonasal dysplasia (Binders syndrome)". Journal of Medical Genetics 27 (1990): 384-387.

4. Nedev P. "The binder syndrome:Review of literature and case report". International Journal of Pediatric Otorhinolaryngology 72 (2008): 1573-1576.

5. Hunter L and Hingston E. "A different look 3 dimensional facial imaging of a child with binder syndrome". American Journal of Orthodontics and Dentofacial Orthopaedics 30 (2010): 201215.

Volume 3 Issue 8 August 2021

(C) All rights are reserved by Prishni Devi Dutta and Swagata Khanna. 